INRA Prod. Anim.,

2012, $25(5), 419-430$

\title{
Conduite et gestion des transitions alimentaires chez les oies destinées à la production de foie gras
}

\author{
J. ARROYO ${ }^{1,2,3,4}$, L. FORTUN-LAMOTHE ${ }^{2,1,3}$, J.-P. DUBOIS 4 , F. LAVIGNE ${ }^{4}$, A. AUVERGNE $E^{1,2,3}$ \\ 1 Université de Toulouse INPT ENSAT, UMR Tissus Animaux, Nutrition, Digestion, Ecosystème et Métabolisme, \\ F-31326 Castanet-Tolosan, France \\ 2 INRA, UMR1289 Tissus Animaux Nutrition Digestion Ecosystème et Métabolisme, \\ F-31326 Castanet-Tolosan, France \\ ${ }^{3}$ Université de Toulouse INPT ENVT, UMR1289 Tissus Animaux Nutrition Digestion Ecosystème et Métabolisme, \\ F-31076 Toulouse, France \\ ${ }^{4}$ ASSELDOR, Station d'Expérimentation Appliquée et de Démonstration sur l'oie, \\ La Tour de Glane, F-24420 Coulaures, France \\ Courriel : laurence.lamothe@toulouse.inra.fr
}

L'élevage des oies destinées à la production de foie gras est réputé pour être techniquement délicat. Un certain nombre de problèmes survenant sur le terrain viennent d'une mauvaise maîtrise de la conduite alimentaire. Les verrous et les leviers d'action permettant d'affronter les périodes sensibles de l'élevage de l'oie destinée à la production de foie gras sont désormais mieux connus.

En France, l'élevage des oies est principalement destiné à la production de foie gras. Par conséquent, le programme d'alimentation des oies en croissance a pour objectif de préparer les oiseaux pour le gavage (Guéméné et Guy 2004). De manière générale, l'élevage de ce palmipède à foie gras peut se décomposer en plusieurs étapes : le démarrage, la croissance-finition et le gavage. De nombreux travaux ont permis de définir un itinéraire technique pour l'élevage (Leprettre et al 1997) et le gavage des oies (Dubois et al 2010), qui optimise les performances des animaux. Cet itinéraire technique comprend plusieurs phases de transitions alimentaires pour s'adapter à l'évolution des besoins nutritionnels des animaux et pour préparer l'animal à l'ingestion d'une quantité très importante d'aliment au cours du gavage. Ces périodes de transitions sont toujours des périodes sensibles et leur gestion conditionne les performances du système d'élevage. Cette synthèse a pour objectif d'identifier les verrous et les leviers d'action à notre disposition pour affronter ces périodes sensibles. Nous rappellerons au préalable l'anatomie, les besoins nutritionnels, les paramètres de la croissance et la conduite alimentaire des oies pour la production de foie gras.

\section{1 / La production d'oie en France et dans le monde}

Actuellement, à travers le monde, les systèmes permettant la production d'oie sont multiples. En effet, les objectifs de production des oies sont variés : viande, foie gras, ou encore production de plumes et de duvet. C'est pourquoi, l'élevage des oies concerne une grande variété d'espèces et de races différentes.

\section{1 / Les races d'oies domestiques utilisées dans le monde}

Plus de 96 races d'oies domestiques ont été décrites et se repartissent principalement dans les pays d'Europe orientale (Guy et Buckland 2002). Les races européennes et asiatiques domestiques proviennent de deux espèces différentes (Anser cygnoides et Anser anser). L'espèce Anser cygnoides est une oie originaire d'Extrême-Orient et la forme domestique est couramment appelée oie de Guinée ou oie de Chine (Anser cygnoides domesticus). L'espèce Anser anser comporte deux sous espèces : l'oie cendrée de l'Ouest (Anser anser anser) et l'oie cendrée de l'Est (Anser anser rubriostris; Kozak et al 1997). Les diverses races se distinguent par la couleur de leur plumage, du bec et des pattes, leur poids à l'âge adulte, leur potentiel de production d'œufs (la saison de ponte dure environ six mois) et le poids des œufs (tableau 1). On y trouve principalement des oies blanches exclusivement destinées à la chair, et des oies grises utilisées aussi bien pour la chair que pour le gavage, tout au moins en France. A l'intérieur de ces groupes, il existe des rameaux ou «souches» qui présentent à leur tour des aptitudes différentes au gavage.

L'oie cendrée sauvage (Anser anser) constitue l'ancêtre commun de toutes les populations d'oies impliquées dans la production de palmipèdes gras dans l'Europe de l'Ouest. D'un poids dépassant les $3 \mathrm{~kg}$ et allant jusqu'à $4,5 \mathrm{~kg}$ pour les mâles, l'oie cendrée sauvage est la plus corpulente des oies indigènes sur le continent eurasiatique. Son poids montre la distance, créée par des siècles de sélection, entre elle et les races domestiques les plus lourdes dépassant les $10 \mathrm{~kg}$ pour l'oie de Toulouse (Dunning 1992). La sélection spécifique pour la production de foie gras n'a été entreprise en France que sur deux sites : la station de l'oie d'Artiguères ${ }^{1}$ à l'initiative

\footnotetext{
1 Actuellement Unité Expérimentale Palmipèdes à Foie Gras (UE PFG).
} 
Tableau 1. Principales souches d'oies domestiques (d'après Guy et Buckland 2002).

\begin{tabular}{|c|c|c|c|c|c|c|c|c|c|}
\hline \multirow{2}{*}{ Espèce $^{1}$} & \multirow{2}{*}{$\begin{array}{l}\text { Souche } \\
\text { ou lignée }\end{array}$} & \multirow{2}{*}{$\begin{array}{c}\text { Couleur } \\
\text { du plumage }\end{array}$} & \multirow{2}{*}{$\begin{array}{l}\text { Couleur } \\
\text { du bec }\end{array}$} & \multirow{2}{*}{$\begin{array}{c}\text { Couleur } \\
\text { des } \\
\text { pattes }\end{array}$} & \multicolumn{2}{|c|}{$\begin{array}{l}\text { Poids vif des } \\
\text { adultes (kg) }\end{array}$} & \multirow{2}{*}{$\begin{array}{c}\text { Nombre } \\
\text { d'œufs } \\
\text { par } \\
\text { ponte }\end{array}$} & \multirow{2}{*}{$\begin{array}{l}\text { Poids } \\
\text { des } \\
\text { œufs } \\
\text { (g) }\end{array}$} & \multirow{2}{*}{$\begin{array}{c}\text { Origine et } \\
\text { répartition } \\
\text { géographique }\end{array}$} \\
\hline & & & & & Mâles & Femelles & & & \\
\hline AA & African & Gris brun & Noir & Orange & 9 & 8 & & & Asie \\
\hline AA & Embden & Blanche & Orange & Orange & 10 & 9 & 40 & 170 & Allemagne \\
\hline AA & Landes & Grise & Orange & Orange & 6 & 5 & $40-45$ & & France \\
\hline AA & Rhin & Blanche & Orange & Orange & 6,5 & 5,5 & 40 & & Allemagne \\
\hline AA & Toulouse & Grise & Orange & Orange & 6,8 & 6,2 & 30 & 160 & France \\
\hline$A C$ & Balien Eu & & & & 4,5 & 3,5 & 125 & 175 & Chine \\
\hline$A C$ & Chine & $\begin{array}{c}\text { Blanche ou } \\
\text { fauve }\end{array}$ & Orange $^{2}$ & Orange & 5,5 & 4,5 & 40 & 120 & Chine \\
\hline$A C$ & Huyon & Blanche & & Orange & 4 & 3,5 & 90 & 120 & Chine \\
\hline$A C$ & Philippines & $\begin{array}{c}\text { Blanche ou } \\
\text { brune }\end{array}$ & & & 2,8 & 2,3 & 50 & & Philippines \\
\hline$A C$ & Rung Eu & & & & 3,5 & 2,5 & 155 & 120 & Chine \\
\hline$A C$ & Wuzhong & Fauve & Noir & Noir & 3,4 & 2,9 & 30 & 145 & Chine \\
\hline
\end{tabular}

${ }_{2}^{1}$ AA : Anser Anser; AC : Anser Cygnoides.

2 sauf le bec de la variété fauve.

du Centre Technique de la Conservation des Produits Agricoles (CTCPA) et de l'Institut National de la Recherche Agronomique (INRA), le Centre de Sélection des Palmipèdes à Foie Gras (SEPALM) à l'initiative des groupements de producteurs :

La station d'Artiguères a utilisé à la base un cheptel d'oies dîtes «Landaises» de petit gabarit dans lequel l'INRA a sélectionné 2 lignées spécialisées : l'une sur les performances de ponte et l'autre sur l'aptitude à produire du foie gras. Actuellement, le travail de sélection est arrêté sur le site.

La SEPALM a généré son cheptel de sélection à partir d'animaux collectés sur le terrain dans tout le sud-ouest, et présentant un gabarit important (oie de

Figure 1. Répartition de la production mondiale de foie gras (canards et oies) en 2011 (27 100 t) (d'après CIFOG 2012).

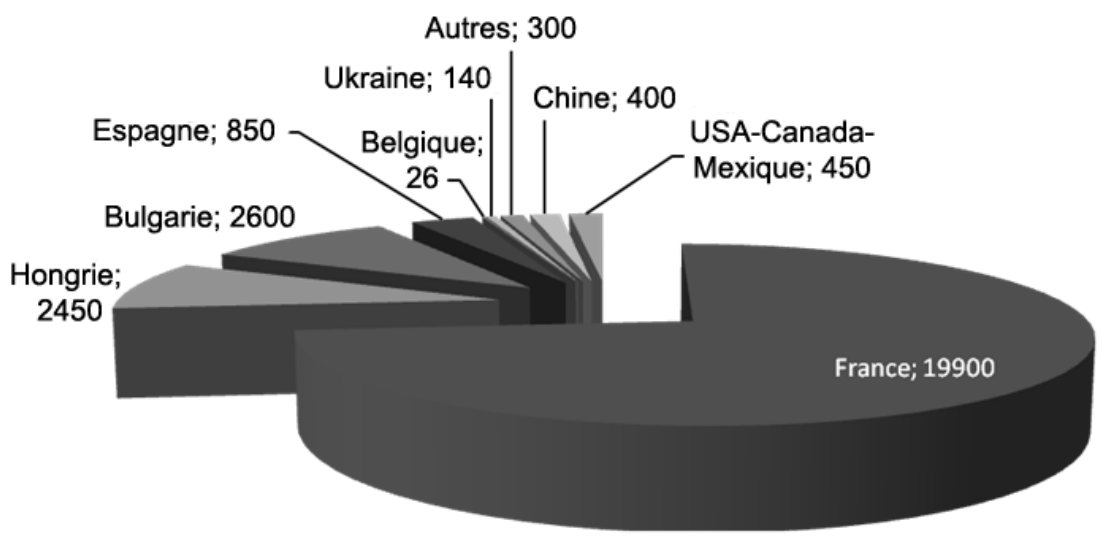

Source : SSP, estimations nationales, Eurofoiegras.
Toulouse, du Gers ou d'origine landaise). Leurs aptitudes de pontes étaient inférieures à celles des animaux sélectionnés par l'INRA.

Les objectifs de sélection étaient proches pour les deux souches : prolificité et poids de foie gras. La sélection pratiquée à l'INRA se distinguait par la recherche d'un taux de viande supérieur (Roussely 2000). Ces souches d'oies sélectionnées ont ensuite été exportées dans de nombreux autres pays européens (Farell 2004).

\section{2 / La production de foie gras en France et dans le monde}

La production mondiale de foie gras est évaluée à 27100 tonnes de foie gras cru en 2011, en progression d'un millier de tonnes par rapport à 2010. Avec environ 25800 tonnes, la production européenne reste très majoritaire au plan international. La France reste le principal pays de production de foie gras avec 19900 tonnes, suivie de la Bulgarie avec 2600 tonnes et de la Hongrie avec près de 2450 tonnes (figure 1, CIFOG 2012).

La production de foie gras de canard domine le marché mondial du foie gras avec environ $90 \%$ du tonnage. Toutefois, c'est la production d'oies grasses qui reste très dominante en Hongrie, Chine et Ukraine. Des ateliers existent dans d'autres pays: Liban, Maroc, Madagascar, Malaisie, etc. La production de ces pays reste très faible, estimée à 300 tonnes, elle est consommée localement (CIFOG 2012).

En France, la production de foie gras d'oie est présente dans quasiment toutes les régions, mais est majoritairement localisée dans le sud-ouest (Aquitaine et Midi-Pyrénées) avec $83 \%$ du cheptel et dans l'ouest de la France (principalement la Bretagne) avec $12 \%$ du cheptel (CIFOG 2012). Cependant, la production de foie gras d'oie d'origine française est aujourd'hui réduite à l'état de niche $(<5 \%$ du tonnage) sur le marché national du foie gras, tant celui-ci est dominé par le foie gras de canard. Une des raisons expliquant cette situation est l'absence de protection juridique pour l'origine géographique du foie gras d'oie qui laisse cette filière démunie face à un afflux massif de produits importés, en provenance notamment de Hongrie et de Bulgarie : 4316 tonnes importées contre 400 tonnes produites en France en 2011 (CIFOG 2012). 
En 2011, la consommation de foie gras mondiale (oie et canard) concerne principalement les pays de l'Union Européenne (UE). La France est le principal pays consommateur de foie gras avec $71 \%$ des tonnages mondiaux consommés (19 597 t). L'Espagne (4143 t), la Belgique (945 t) et l'Allemagne (199 t) sont les principaux autres pays consommateurs de l'UE. Le Japon (779 t), la Suisse (412t), la Chine (400 t) et les Etats-Unis $(380 \mathrm{t})$ sont les principaux pays consommateurs de foie gras à l'extérieur de l'UE.

\section{2 / Anatomie et physiologie digestive}

Le tractus digestif de l'oie est proche de celui des autres espèces aviaires granivores (figure 2) à l'exception de trois particularités propres aux volailles herbivores (voir ci-après). L'œsophage de l'oie est relativement long, il possède des glandes à mucus qui lubrifient les aliments et facilitent leur passage. Il s'élargit en forme de fuseau pour constituer un réservoir à nourriture : le jabot. Les aliments passent ensuite dans le proventricule dont la fonction principale est la sécrétion gastrique (acidification, pepsine). La fonction du gésier est d'abord mécanique (broyage des graines) et ensuite digestive : c'est à ce niveau que débute la dégradation des protéines. L'intestin est le site principal de la digestion où interviennent les sécrétions de bile, de sucs pancréatiques et intestinaux. La fermentation microbienne des fibres alimentaires intervient dans les caeca sur une partie des digesta. Mais la majeure partie passe directement vers le cloaque où sont excrétés à la fois les urines et les fèces comme chez tous les oiseaux. Le tractus digestif de l'oie possède trois particularités : i) le jabot est peu développé, à la différence de celui du canard ou de la poule (Sturkie 1986), ii) le gésier est extrêmement musclé et peut développer des pressions atteignant $275 \mathrm{~mm}$ de mercure (vs 180 et $125 \mathrm{~mm}$ chez le canard et la poule, respectivement), iii) les caeca sont très développés (Jamroz et al 2001).

L'utilisation d'aliments riches en fibres est rendue possible par $i$ ) leur dégradation physique dans le gésier (Moore 1999) et ii) leur fermentation microbienne dans les caeca (Yang et al 2009). La digestibilité des fibres alimentaires chez l'oie va de 18 à $46 \%$ et dépend de la nature des fibres ingérées (Hsu et al 1996). On peut noter que cette dégradation assez importante intervient en dépit du fait que le transit digestif est relativement rapide chez l'oie (Guy et Buckland 2002).

Figure 2. Tractus digestif de l'oie (d'après Clemens et al 1975, Guy et Buckland 2002).
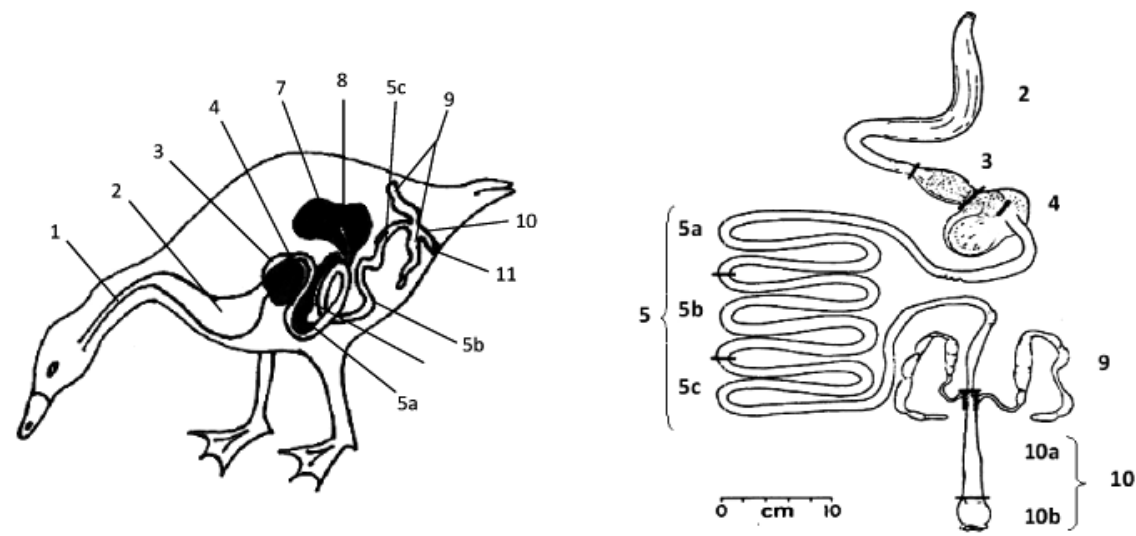

1. OEsophage ; 2. Jabot ; 3. Proventricule ; 4. Gésier ; 5. Intestins, 5a. Ventricule proximal (duodénum), 5b. Ventricule médian (jéjunum), 5c. Ventricule distal (iléon); 6. Pancréas ; 7. Foie ; 8. Vésicule biliaire ; 9 . Caecas; 10. Colon, 10a. Rectum, 10b. Cloaque; 11. Anus.

Le travail de concassage des aliments dans le gésier est permis par la présence de gravillons qui sont consommés de façon naturelle par les oiseaux. En élevage, ces gravillons (dénommés «grit» ou «pierres d'estomac») doivent être mis à la disposition des animaux en quantité suffisante $(1 \mathrm{~kg} / 100$ oies/semaine $)$ pour permettre une utilisation optimum des ressources alimentaires.

\section{3 / La croissance des oies destinées à la production de foie gras}

L'oie est l'une des espèces de volailles à la croissance la plus rapide dans le monde (Leeson et Summers 1997). La croissance et le dépôt protéique des oisons est très rapide, particulièrement durant les quatre premières semaines de vie. En effet, le plumage destiné à la protection face à l'eau et au froid est particulièrement développé chez les oies. D'ailleurs, les protéines retenues dans les plumes chez l'animal adulte sont élevées $(85 \mathrm{~g}$ de protéines $/ \mathrm{kg}$ de poids corporel). Entre 2 et 4 semaines d'âge, le taux de protéines conservé dans les plumes est de 33 contre 58\% dans la carcasse (Nitsan et al 1981). Dubois et al (1996a) ont montré que la croissance pondérale initiale est très forte et que les animaux atteignent en moyenne $4,9 \mathrm{~kg}$ pour les mâles et $4,3 \mathrm{~kg}$ pour les femelles à l'âge de 8 semaines (poids d'un oison à l'éclosion : $100 \mathrm{~g}$ ) soit une vitesse de croissance moyenne de $80 \mathrm{~g} / \mathrm{j}$. Dans les systèmes d'élevage destinés à la production de foie gras, sous l'effet du rationnement mis en œuvre pour préparer le gavage (cf. $\S$ 5.2 ), le gain de poids s'infléchit notablement après 8 semaines. Ceci se traduit par une stabilité de l'écart de poids entre les mâles et les femelles $(+0,62 \mathrm{~kg}$ en moyenne chez les mâles). L'écart de croissance entre les sexes semble lié à une différence de format, sans qu'il y ait de différence notable de la vitesse de croissance (figure 3 ).

Dubois et al (1996a) ont montré que le muscle pectoral a un développement tardif et maximum entre 6 et 12 semaines d'âge $(50$ et $280 \mathrm{~g}$ à 6 et 14 semaines d'âge chez les mâles, respectivement). A l'opposé, le membre inférieur se développe précocement et n'évolue pratiquement plus après la $8^{\text {eme }}$ semaine $(388 \mathrm{~g}$ pour la cuisse-pilon sans peau chez les mâles). Le développement des tissus adipeux intra-abdominal et périphérique est continu entre 6 et 14 semaines d'âge et peu affecté par la méthode de rationnement (1067 g à 14 semaines chez les mâles, Dubois et al 1996a). L'allométrie de croissance est similaire entre les sexes pour tous les tissus évoqués ci-dessus.

\section{4 / Les besoins nutritionnels et énergétique des oies}

Comme pour tous les animaux d'élevage, l'équilibre de la ration alimentaire des oies est primordial pour en assurer une valorisation efficace et permettre une croissance optimale des animaux. L'étude des besoins nutritionnels de l'oie pour sa croissance a généralement été décomposée en trois phases (tableau 2) : la première allant de 0 à 2 semaines d'âge, la seconde de 3 à 5 semaines et la troisième de 6 à 14 semaines. Les données de la bibliographie sur les besoins nutritionnels des oies sont contradictoires (Daghir 2008). Ces contradictions peuvent s'expliquer par les différences de format des animaux entre les souches, les objectifs de production (foie gras, plume, viande, ouf) et les conditions environnementales. Nous proposons une synthèse de travaux les plus cohé- 
Figure 3. Courbe de croissance et de consommation d'oie mâle et femelle (d'après Arroyo et al 2012b).

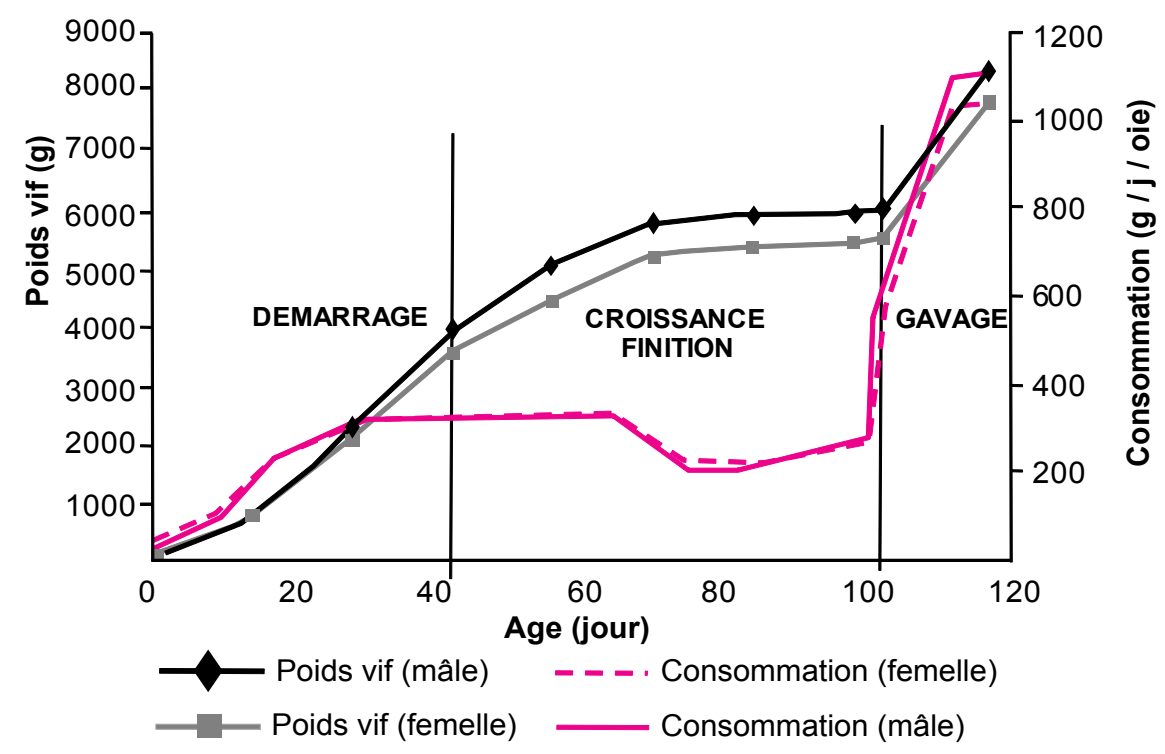

rents pour la souche utilisée pour la production de foie gras c'est-à-dire la souche «Landaise». Toutefois, il faut noter que les oies sont élevées en système semiplein air, par conséquent les valeurs présentées ci-dessous sont des valeurs médianes et peuvent fluctuer de $\pm 5 \%$ suivant la saison.

\section{1 / Energie et protéines}

Les besoins en Energie Métabolisable (EM) et en protéines pendant la première phase sont de $2900 \mathrm{kcal} \mathrm{EM} / \mathrm{kg}$ et $21 \%$. Au cours de la seconde phase, les besoins en énergie et en protéines diminuent $(2800 \mathrm{kcal} \mathrm{EM} / \mathrm{kg}$ et $18 \%$; Auvergne et al 2012). Enfin, au cours de la troisième phase, les besoins en énergie stagnent $(2800 \mathrm{kcal} \mathrm{EM} / \mathrm{kg}$ ) tandis que les besoins en protéines continuent à diminuer $(17 \%)$.

\section{2 / Acides aminés}

Comme pour les autres volailles, les principaux acides aminés limitants dans l'alimentation des oies sont les acides aminés soufrés (méthionine et cystine), la lysine, le tryptophane et la thréonine. Les recommandations concernant les besoins en acides aminés soufrés pendant la première phase sont de $0,82 \mathrm{~g} / 100 \mathrm{~g}$ d'aliment. Au cours de la seconde et de la troisième phase, les besoins diminuent (respectivement 0,58 et $0,50 \mathrm{~g} / 100 \mathrm{~g}$ d'aliment; Leclercq et al 1987, NRC 1994, Leeson et Summers 1997).

\section{3 / Minéraux et vitamines}

Les besoins pour les principaux minéraux et vitamines sont présentés dans le tableau 2. Les minéraux les plus limitants sont le calcium et le phosphore comme pour les autres volailles. Leclercq et al (1987) indiquent que pendant les trois premières semaines de vie des animaux, les besoins en calcium et en phosphore disponibles sont respectivement de $0,78 \mathrm{~g} / 100 \mathrm{~g}$ d'aliment et de

Tableau 2. Concentrations recommandées en énergie, protéines brutes, acides aminés, minéraux et vitamines des aliments pour les oisons en croissance (d'après Leclercq et al 1987, NRC 1994, Auvergne et al 2012).

\begin{tabular}{|l|c|c|c|}
\hline & $\mathbf{0 - 2}$ semaines & 3-5 semaines & $\mathbf{6 - 1 4}$ semaines \\
\hline Energie (kcal EM / kg d'aliment) & 2900 & 2800 & $\mathbf{2 8 0 0}$ \\
\hline Protéines brutes (g / 100 g d'aliment) & 21 & 18 & 17 \\
\hline Acides aminés (g / 100 g d'aliment) & & & 0,49 \\
\hline Lysine & 0,92 & 0,58 & 0,26 \\
\hline Méthionine & 0,41 & 0,30 & 0,50 \\
\hline Acides aminés soufrés & 0,82 & 0,58 & 0,13 \\
\hline Tryptophane & 0,18 & 0,14 & 0,45 \\
\hline Thréonine & 0,60 & 0,48 & \\
\hline Minéraux (g / 100 g d'aliment) & & & 0,68 \\
\hline Calcium & 0,78 & 0,78 & 0,59 \\
\hline Phosphore total & 0,69 & 0,64 & 0,34 \\
\hline Phosphore disponible & 0,44 & 0,39 & 0,15 \\
\hline Sodium & 0,15 & 0,15 & 0,14 \\
\hline Chlorures & 0,14 & 0,14 & 1500 \\
\hline Vitamines (Ul / kg d'aliment) & & & 200 \\
\hline Vitamines A & 1500 & 1500 & \\
\hline Vitamines $\mathrm{D}_{3}$ & 200 & 200 & 1000 \\
\hline Vitamines (mg / kg d'aliment) & & & 35 \\
\hline Choline & 1500 & 1000 & 2,0 \\
\hline Niacine & 65 & 35 & 10,0 \\
\hline Acide pantothénique & 15,0 & 2,5 & \\
\hline Riboflavine & 3,8 & & \\
\hline
\end{tabular}


0,44 g/100 g d'aliment. Par la suite, ces besoins diminuent pour être respectivement de $0,68 \mathrm{~g} / 100 \mathrm{~g}$ d'aliment et $0,34 \mathrm{~g} / 100 \mathrm{~g}$ d'aliment pour le calcium et le phosphore disponible, respectivement entre 7 et 12 semaines.

D'après le NRC (1994), les recommandations nutritionnelles en vitamines sont de 1500 et $200 \mathrm{UI} / \mathrm{kg}$ d'aliment respectivement pour les vitamines A et $\mathrm{D}_{3}$ pendant toute la croissance des animaux.

\section{5 / Elevage et conduite ali- mentaire}

\section{1 / La conduite d'élevage}

De manière générale, l'oie est un animal résistant et peut être élevé en plein air avec un minimum d'abri et de soins. A travers le monde, il y a beaucoup de petits troupeaux d'oies libres dans de petites exploitations, qui ont souvent pour simple confort, un étang et un refuge contre les renards. Par exemple, en Chine, les oies sont gérées, quelle que soit la taille du troupeau, avec la liberté de sortir de leur bâtiment. De plus, l'oie est un animal «pâtureur» qui valorise les fourrages. Elle est capable d'ingérer, à l'âge adulte, plus de $1200 \mathrm{~g}$ de verdure par jour (Leprettre et al 2000a). L'oie préfère les graminées et consomme peu les feuilles de dicotylédones probablement en raison d'une mauvaise dégradation physique de ces feuilles dans le gésier (Moore 1999). Dubois et al (2008) ont montré que le dactyle (Dactylis glomerata) permet de mieux concilier la consommation des animaux et la résistance du couvert végétal au piétinement des animaux que la fétuque (Festuca arundinacea), le brome (Bromus hordeaceus) ou le ray-grass anglais (Lolium perenne). C'est pourquoi, l'oie peut se contenter d'un minimum voire de pas du tout d'apport d'aliment complémentaire (Farell 2004). Toutefois, la productivité des animaux sera évidemment réduite en cas d'apports nutritionnels limités. En conséquence, en élevage rationnel, l'alimentation des oies n'est pas basée sur les fourrages mais repose sur l'utilisation d'aliments concentrés. Le fourrage vert constitue alors une petite partie des apports lorsque les animaux ont accès à un parcours enherbé.

En Europe, particulièrement en Hongrie et en France, pour produire du foie gras les oies sont élevées dans des systèmes intensifs sur litière accumulée (paille), sur caillebotis ou en plein air. Les bâtiments peuvent être entièrement fermés avec un accès extérieur clôturé (Farell 2004).
Pendant la phase de démarrage, les oisons sont, en général, élevés en claustration pour faciliter la maîtrise des conditions de température qui sont nécessaires à leur développement (Dubois et al 2006, Auvergne et al 2008b, Auvergne et al 2010). L'objectif est qu'il n'y ait pas de choc thermique entre l'ambiance de l'éclosoir et celle du bâtiment d'élevage. Pour ce faire, il convient d'allumer le chauffage au moins 24 heures avant l'arrivée des oisons. La température ambiante doit être maintenue à $28^{\circ} \mathrm{C}$ pendant la première semaine après l'éclosion et peut ensuite être progressivement réduite pour atteindre $22^{\circ} \mathrm{C}$ à 7 semaines d'âge, après quoi la fourniture de chaleur n'est plus nécessaire (Auvergne et al 2008b). Un radian (4500 mth) pour 150 oisons permet de fournir la chaleur nécessaire au confort thermique des animaux (Dubois et al 1998). La réussite d'une bande d'oies dépend en grande partie du succès de cette première phase qui repose sur les soins prodigués et sur l'attention portée aux jeunes oisons (Guy et Buckland 2002). Après 7 semaines d'âge, les animaux ont généralement accès à un parcours extérieur enherbé (10 m²/oie).

En élevage rationnel, la densité des animaux dans les bâtiments est plus élevée que dans les systèmes extensifs. On recommande généralement une densité de 5 oies $/ \mathrm{m}^{2}$ au cours de la phase de démarrage et jusqu'à 6 semaines d'âge. Cette densité élevée peut être pratiquée jusqu'à la mise en gavage si les autres conditions d'élevage sont favorables (accès à l'eau, à l'aliment, confort thermique...) (Leprettre et al 2000b). Cependant, travailler avec des densités de 5 oies $/ \mathrm{m}^{2}$ nécessite un entretien plus important des litières, pour limiter leur humidification, sous peine d'avoir des animaux présentant des défauts d'emplumement au niveau ventral, au niveau du bréchet et des filets, source de déclassement des carcasses à l'issue du gavage. Il est donc recommandé de réduire la densité d'élevage à 3,5 oies $/ \mathrm{m}^{2}$ de 6 semaines d'âge jusqu'à la mise en gavage.

\section{2 / La conduite alimentaire}

Afin de stimuler la synthèse des lipides et le stockage lipidique dans le foie, caractéristique propre à certains palmipèdes dont les oies, les producteurs ont développé des programmes d'alimentation spécifiques adaptés à la production de foie gras. Le plan d'alimentation comporte 3 phases distinctes : la phase de démarrage (de 1 à 6 semaines d'âge), la phase de croissance et finition (de 7 à 14 semaines d'âge), et la phase de gavage (de 15 à 16 semaines d'âge, tableau 3 ). La consommation de l'oie évolue au cours de leur croissance. Elle est d'environ $70 \mathrm{~g}$ d'aliment par jour pour un aliment complet équilibré $(2800 \mathrm{kcal} / \mathrm{kg}$ d'énergie métabolisable, $20 \%$ de protéines) au cours de la première semaine de vie, et augmente progressivement pour atteindre près de $400 \mathrm{~g} / \mathrm{j}$ (2800 kcal/kg d'énergie métabolisable, $17 \%$ de protéines).

\section{a) Démarrage}

Les objectifs et les enjeux de cette première phase sont de bien stimuler la phase d'alimentation précoce pour entamer la croissance et ainsi favoriser l'homogénéité des lots. Durant les deux premières semaines de leur vie, les animaux reçoivent ad libitum une alimentation sous forme de miettes. A partir de la troisième semaine d'âge, les oies reçoivent des aliments sous forme de petits granulés (par exemple : $3 \mathrm{~mm}$ de diamètre et $8 \mathrm{~mm}$ de longueur).

\section{b) Croissance et finition}

Les objectifs et les enjeux de cette seconde phase sont de terminer la croissance des animaux tout en les préparant à l'alimentation forcée qui sera pratiquée pendant la phase de gavage.

Durant cette période les oisons reçoivent des aliments sous forme de granulés complets équilibrés (par exemple $4 \mathrm{~mm}$ de diamètre et $9 \mathrm{~mm}$ de longueur). Mais, tout comme chez le poulet, une alimentation composée d'un mélange de graines entières (le plus souvent une céréale) et d'un complément granulé riche en protéines peut être réalisée. Cette stratégie d'alimentation peut être effectuée soit de manière séquentielle, ces deux fractions sont alors distribuées à deux moments différents de la journée (Bouvarel et al 2008, Umar Faruk et al 2010), soit en libre choix dans deux mangeoires différentes (Henuk et Dingle 2002, Pousga et al 2005), soit encore sous forme de mélange dans une même mangeoire (Yo et al 1997, Arroyo et al 2012a et b). L'accès à un parcours enherbé pendant la phase de croissance et finition contribue de manière significative aux apports alimentaires (Dubois et al 2008, Bijja et al 2010). Les apports d'aliment concentré doivent être adaptés en fonction de la contribution du parcours herbagé aux apports nutritionnels. Romanov (1999) n'a rapporté aucune différence dans le poids final des oies (24 semaines d'âge) lorsqu'elles étaient nourries soit avec un aliment concentré (220-250 g/oie/j, $2200 \mathrm{kcal} / \mathrm{kg}$ d'aliment) seul sans accès à un pâturage, soit avec du maïs (130$150 \mathrm{~g} / \mathrm{oie} / \mathrm{j}, 3860 \mathrm{kcal} / \mathrm{kg}$ ) et un accès à un pâturage ( $8 \%$ trèfle, $46 \%$ de raygrass et $46 \%$ de fétuque).

Afin de préparer les animaux à l'alimentation forcée, un système de rationnement est mis en place à la fin de la phase de croissance et finition. Ce rationnement 
Tableau 3. Conduite alimentaire des oies destinées à la production de foie gras (d'après Auvergne et al 2012).

\begin{tabular}{|c|c|c|c|}
\hline Semaines & Période & $\begin{array}{l}\text { Conduite alimentaire } \\
\text { «rationnement horaire» }\end{array}$ & Aliment \\
\hline 1 & \multirow{5}{*}{ Démarrage } & Ad libitum & \multirow{2}{*}{ Miettes } \\
\hline 2 & & Ad libitum & \\
\hline 3 & & Ad libitum & \multirow{3}{*}{ Granulé complet } \\
\hline 4 & & Ad libitum & \\
\hline 5 & & Ad libitum & \\
\hline 6 & \multirow{9}{*}{ Croissance-finition } & Ad libitum & \multirow{9}{*}{$\begin{array}{l}\text { Granulé complet } \\
\text { ou } \\
\text { Céréale + Granulé complémentaire }\end{array}$} \\
\hline 7 & & Ad libitum & \\
\hline 8 & & 12 h/jour (la nuit) & \\
\hline 9 & & $3 \mathrm{~h} /$ jour & \\
\hline 10 & & $2 \mathrm{~h} /$ jour & \\
\hline 11 & & $2 \mathrm{~h} /$ jour & \\
\hline 12 & & $2 \mathrm{~h} /$ jour & \\
\hline 13 & & $2 \mathrm{~h} /$ jour & \\
\hline 14 & & $3 \mathrm{~h} /$ jour & \\
\hline 15 & \multirow{2}{*}{ Gavage } & \multirow{2}{*}{2 à 5 repas par jour } & \multirow{2}{*}{$\begin{array}{l}\text { Maïs } \\
\text { ( } 42 \% \text { graines entières et } 52 \% \text { de farine) }\end{array}$} \\
\hline 16 & & & \\
\hline
\end{tabular}

est dit «rationnement horaire» car ce sont les temps d'accès à la mangeoire qui sont restreints. Ainsi, à partir de la $9^{\text {eme }}$ semaine d'âge, le temps d'accès à la mangeoire est restreint à la période nocturne. Puis, de 10 à 11 semaines d'âge, l'accès à l'aliment est restreint à $2 \mathrm{~h}$ par jour. Enfin, durant les quatre derniers jours avant la mise en gavage, les oies n'ont accès à la mangeoire que $3 \mathrm{~h} /$ jour (Dubois et al 1996b, Arroyo et al 2012a). Cette augmentation de la durée du repas au cours des 3-4 jours qui précédent le gavage a pour objectif d'accentuer le développement du jabot et de faciliter le gavage (Leprettre et al 1997, Guy et al 1998). Cette pratique permet en effet une augmentation significative de la quantité d'aliment ingérée (de $200 \mathrm{~g} / \mathrm{j}$ jusqu'à $400 \mathrm{~g} / \mathrm{j}$ ). Toutefois, les niveaux d'ingestion sont fortement dépendants des conditions environnementales. Ainsi, l'ingestion chute lorsque les températures sont élevées (supérieures à $25^{\circ} \mathrm{C}$, Meltzer 1987). Un rationnement quantitatif (maîtrise des quantités distribuées) peut également être mis en place pour remplacer ou compléter le rationnement horaire. Celui-ci est moins fréquent car plus délicat à mettre en œuvre. En effet, il suppose de réaliser en permanence un calcul de ration hebdomadaire en fonction des conditions ambiantes et une surveillance de la durée du repas pour permettre une préparation efficace du jabot. Un rationnement trop sévère se traduira par un poids des animaux à la mise en gavage trop faible ainsi qu'une réduction du poids et de la qualité du foie et des magrets (Dubois et al 1996b).

\section{c) Gavage}

Les objectifs et les enjeux de cette dernière phase sont de stimuler la synthèse des lipides et les capacités de stockage dans le foie et dans les tissus sous-cutanés. Son principe réside dans la consommation excessive de céréales riches en amidon qui fournit une alimentation hyper énergétique. Cette surconsommation d'hydrates de carbone induit la synthèse d'acides gras par le tissu hépatique. Parce que le maximum de la capacité d'exportation de ces acides gras est rapidement atteint, les lipides sont alors stockés dans le foie qui devient de plus en plus gras (Hermier et al 1999). La mise en gavage des oies a lieu après la $14^{\text {ème }}$ semaine, alors que les femelles pèsent en moyenne $5,5 \mathrm{~kg}$ et les mâles $6 \mathrm{~kg}$ (Rouvier et al 1992). Afin d'obtenir de meilleures performances durant cette phase, il est souhaitable $\mathrm{d}$ 'avoir des lots homogènes d'animaux et d'adapter le rythme de gavage et les quantités distribuées à la souche, au développement des animaux et aux objectifs de production (Lavigne et al 2000, Dubois et al 2010).

La pâtée de gavage distribuée est composée de $590 \mathrm{~g}$ de maïs/kg de pâtée dont $248 \mathrm{~g}$ de graine entière et $342 \mathrm{~g}$ de farine auxquelles sont ajoutés $390 \mathrm{~g}$ d'eau / $\mathrm{kg}$ de pâtée et $20 \mathrm{~g}$ d'un complément minéral et vitaminique $/ \mathrm{kg}$ de pâtée (Arroyo et al 2012c).

Actuellement, le gavage des oies est réalisé pendant une durée de 14 à 18 jours et permet d'obtenir des foies de
$850 \mathrm{~g}$ en moyenne (poids recherché par les conserveurs). Les recommandations sont de 2,3 à 2,4 $\mathrm{kg}$ de maïs $/ \mathrm{kg}$ de poids vif à la mise en gavage pour des gavages de 14 à 16 jours et 2,7 à 3,0 de maïs $/ \mathrm{kg}$ de poids vif à la mise en gavage pour des gavages en 18 jours. Les quantités d'aliment distribuées pendant cette période sont plus faibles pour les femelles que pour les mâles en raison de leur poids plus léger, avec respectivement $12,8 \mathrm{~kg}$ et $14 \mathrm{~kg}$ d'aliment au total (figure 3). Le maïs reste le principal ingrédient utilisé pour le gavage, parce que c'est un ingrédient de faible coût qui présente une teneur élevée en amidon. Or, l'amidon est un des meilleurs substrats pour stimuler la lipogenèse dans le foie des oiseaux (Guéméné et Guy 2004). De nombreuses autres matières premières, riches en amidon (sorgho : Arroyo et al 2012c), en protéines (soja : Nir et al 1972), en glucides non amylacés (figue : Babilé et al 1998), ou en lipides (tourteau de noix : Bouiller-Oudot et al 2002) ont également été testées pour stimuler la stéatose hépatique. Parmi ces études, seul le gavage au sorgho, dont la composition nutritionnelle est très proche de celle du maïs (Sauvant et al 2004) a donné de meilleurs résultats pondéraux que le gavage au maïs (Arroyo et al 2012c). Toutefois, l'utilisation du sorgho modifie la couleur du foie gras (foies plus pâles) en raison de la teneur moins élevée en caroténoïdes du sorgho que celle du maïs. Des cas de mortalité pendant le gavage ont également été observés ce qui montre qu'il faut sans doute adapter un peu la conduite alimentaire à l'utilisation de cette céréale. 
En raison de sa disponibilité et de son aire géographique de production, qui se superpose avec le bassin de production du foie gras, le maïs reste la principale matière première utilisée pour le gavage des palmipèdes.

\section{6 / Facteurs affectant les performances des animaux au moment des transitions alimentaires}

Afin de préparer les oies à recevoir une forte quantité d'aliments dans une période courte (gavage), celles-ci sont soumises tout au long de leur vie à des changements d'aliments et à des rythmes d'accès aux mangeoires évolutifs afin d'augmenter leur capacité d'ingestion. Lors de chacune de ces phases de transitions, on observe fréquemment des baisses de consommation, ce qui entraîne une hétérogénéité des animaux au sein d'un même lot, et une réduction des performances des animaux. La gestion de ces transitions vient se confronter aux capacités d'adaptation comportementale et digestive des animaux face à des modifications des caractéristiques sensorielles et physico-chimiques des aliments.

\section{1 / Effets de la forme de présen- tation et de l'aspect de l'aliment}

La première transition alimentaire survient à l'âge de 2 semaines et correspond au passage d'un aliment sous forme de miettes à l'aliment de «démarrage», présenté sous forme de granulés de petite taille. Cette transition n'engendre généralement pas de difficultés ni de troubles chez les animaux. La seconde transition alimentaire survient à la $6^{\text {ème }}$ semaine d'âge et correspond au passage de l'aliment de «démarrage» à l'aliment dit de «croissance-finition» qui est généralement présenté sous forme d'un granulé complet de taille supérieure à celle du granulé distribué pendant le démarrage (Arroyo et al 2012b) ou d'un mélange de graines entières (de diamètre compris entre $3 \mathrm{~mm}$ pour du sorgho à plus de $4 \mathrm{~mm}$ pour du maïs) et de granulés riches en protéines (Arroyo et al $2012 b)$. Lors de cette transition, on observe fréquemment chez les palmipèdes une baisse de la consommation (- 33\% ; Arroyo et al 2012a). Plusieurs éléments peuvent expliquer ce phénomène et des solutions pratiques existent pour diminuer ces effets.

La consommation d'aliment est influencée par les besoins de l'individu et par ses préférences alimentaires (Bouvarel et al 2010). On peut distinguer deux types de régulation de l'ingestion des aliments : à long terme, l'ingestion est régulée au niveau central par des boucles de rétroaction issues des tissus périphériques, permettant d'assurer l'équilibre énergétique, l'homéostasie protéique et l'homéothermie ; à court terme, l'ingestion est régulée par la nécessité de limiter l'encombrement de l'aliment ingéré et d'assurer des apports réguliers d'énergie et de nutriments. L'ingestion fait intervenir des signaux provenant du tractus gastro-intestinal et agissant au niveau central. Les signaux sensoriels conditionnent la réponse à très court terme (inférieure à la minute). Parmi eux, les capacités visuelles et tactiles sont particulièrement utilisées par les volailles pour apprécier leur aliment. Le comportement alimentaire dépend de ces différentes perceptions qui interagissent entre elles et avec l'expérience de l'animal (Bouvarel et al 2010).

Ainsi, au cours d'une alimentation mélangée (céréales entières et granulés riches en protéines), les oies en croissance réalisent un tri des aliments et consomment préférentiellement les granulés riches en protéines au début de la phase de distribution. Cette tendance s'inverse au profit des céréales entières vers l'âge de 11 semaines (Arroyo et al $2012 \mathrm{a}$ et $\mathrm{b}$ ). Ce phénomène peut être expliqué par le fait que les oiseaux équilibrent spontanément leur régime alimentaire selon leurs besoins protéiques qui diminuent avec l'âge (Hughes 1984). Ce phénomène a également été observé chez les poulets de chair recevant des céréales entières (Emmans 1991, Covasa et Forbes 1994, Forbes et Shariatmadari 1994).

Comme chez les autres volailles, il a été montré que la consommation d'aliment par les oies peut être influencée par la couleur (Roper et Marples 1997, Lecuelle et al 2010), la texture (Bouvarel et al 2009), la taille (Auvergne et al 2008a; Arroyo et al 2012a) et/ou la flaveur (Capretta et Moore 1970) de la nourriture. L'oie est un animal grégaire dont la prise alimentaire est influencée par le comportement du groupe. Ainsi, la facilitation sociale (Clayton 1978) et l'allélomimétisme alimentaire (Hughes 1971, Keeling et Hurnik 1996), c'est-àdire l'augmentation de la fréquence ou de l'intensité des réponses ou de l'initiation de réponses particulières dues à la présence d'autres animaux engagés dans un comportement, vont entraîner une consommation ou au contraire une néophobie du groupe vis-à-vis des aliments (Nielsen 2004). Toutefois, le déterminisme de la prise alimentaire reste peu connu chez l'oie (Marcilloux et Auffray 1981, Ramseyer et al 2009).

L'utilisation de différentes matières premières (triticale, pois, féverole, lupin :
Leprettre et al 2000a, Auvergne et al 2006 ; maïs : Auvergne et al 2008a, Arroyo et al 2012b; sorgho: Arroyo et al 2012a et b) présentées sous des formes variées (granulés, farines plus ou moins grossières, graines concassées, graines entières) a permis de montrer que les jeunes oies consomment plus facilement les particules de taille comprise entre $2 \mathrm{~mm}$ et $4 \mathrm{~mm}$. Ce n'est qu'à partir de 8 semaines d'âge qu'elles consomment des particules de taille supérieure à $4 \mathrm{~mm}$. En conséquence, l'utilisation du maïs sous forme de graines entières est à éviter avant cet âge et doit être remplacée par celle du blé, du triticale ou du sorgho. En revanche, le maïs peut être utilisé sous forme aplatie ou concassée. Il conviendra toutefois de limiter la présence de particules fines dans le mélange pour éviter le gaspillage.

Afin de réduire au maximum la chute de consommation au moment des changements d'aliment, il convient de réaliser pendant une semaine un mélange des aliments des deux phases dans la mangeoire. La transition ainsi progressive, laissera le temps nécessaire à l'animal pour s'adapter et permettra de limiter la chute de performance. Arroyo et al (2012b) ont observé que les effets d'un changement d'aliment sont dépendants de l'âge, donc du poids et de l'expérience passée des animaux, au moment de la transition. Ainsi, une transition plus tardive entre l'aliment de démarrage et de croissance, à 8 au lieu de 7 semaines d'âge, affecte moins l'ingestion et les performances de croissance des oies. En conséquence, pour limiter leurs effets, les transitions alimentaires devraient être adaptées au développement des animaux.

Comme cela a été démontré chez la volaille (Forbes et Covasa 1995, Noirot et al 1998, Carré 2000, Amerah et al 2007, Mirghelenj et Golian 2009), la forme physique de l'aliment distribué pendant la phase d'élevage affecte le développement des différentes parties du tractus digestif des animaux (Lu et al 2011, Arroyo et al 2012a et b). Ainsi, Arroyo et al (2012b) montrent que l'utilisation de graines entières tend à augmenter le développement des intestins $(+9 \%)$, par rapport à l'utilisation de granulés complets. Cependant, malgré la mise en place du rationnement, le volume du jabot n'augmente que de manière partielle avec l'utilisation des graines entières. Par exemple, Arroyo et al (2002b) montrent que le volume du jabot est plus petit $(-10 \%)$, chez les oiseaux nourris avec un mélange de granulés protéiques et de graines entières que chez ceux nourris avec des granulés complets. Ces résultats peuvent être expliqués par une plus faible capacité d'hydratation des graines entières que 
des granulés dans lesquels les graines ont été préalablement broyées. En effet, la forme de présentation d'une matière première modifie sa capacité d'hydratation. Les processus de broyage et de granulation réduisent la taille des particules et augmentent leur surface de contact avec l'eau ; de plus, la granulation gélatinise partiellement l'amidon, ce qui accroît également sa capacité d'hydratation (Pinnavaia et Pizzanrani 1998).

Il a été montré que la capacité d'hydratation d'un aliment augmente avec sa teneur en parois cellulaires (Robertson et Eastwood 1981, Buffo et al 1998, GigerReverdin 2000) et dépend de la nature de l'amidon (Pinnavaia et Pizzanrani 1998). L'incorporation à un taux élevé de matières premières à forte capacité d'hydratation dans l'alimentation des oies au cours de la phase de finition semble une perspective très intéressante pour augmenter le volume du jabot et préparer les animaux au gavage.

\section{2 / Effets du mode de distribu- tion de l'aliment}

Un épisode de prise alimentaire comporte trois phases : une phase pré-ingestive caractérisée par la sensation de faim et la recherche active d'aliment, une phase prandiale correspondant à la période de prise alimentaire et à la mise en place progressive du rassasiement, et une phase post-prandiale caractérisée par l'état de satiété dont la durée est variable (Blundell 1991). La régulation des apports alimentaires peut se faire à la fois sur la quantité et la qualité des aliments ingérés au cours d'un épisode de prise alimentaire, ce qui met en jeu le processus de rassasiement, et sur la durée de l'intervalle entre deux prises alimentaires (Savory 1999). De plus, la motricité gastro-intestinale, adaptée à un certain rythme d'ingestion, a besoin de délais d'adaptation lors de changement de rythmes alimentaires (Duke 1982, 1992). On ne dispose cependant que de peu de données sur la capacité d'adaptation du tube digestif aux transitions alimentaires. L'alimentation par repas (accès quotidien à la mangeoire réduit) a pour objectif d'habituer les animaux à ingérer leur ration journalière en un temps restreint et de les préparer à la phase de gavage. La transition entre une alimen- tation ad libitum et une alimentation par repas ( 2 heures d'accès par jour) conduit à une réduction des quantités consommées d'environ $15 \%$. Après une à deux semaines d'adaptation et en absence de changement d'aliment, on retrouve une consommation quotidienne similaire à celle observée avec un accès libre à la mangeoire (Arroyo et al 2012a).

La prise en compte du rythme de la prise alimentaire semble être une perspective intéressante dans la gestion de la mise en place de l'alimentation par repas ainsi que du passage de la phase d'élevage à la phase de gavage. Ainsi, synchroniser les heures d'accès à la mangeoire avec le rythme d'ingestion naturel et/ou celui prodigué pendant le gavage, sans modifier la durée totale d'accès, pourrait permettre de limiter les digestions difficiles qui sont fréquemment rencontrées en début de gavage et qui entraînent des non distributions de repas. Ce phénomène affecte par la suite l'homogénéité du lot ainsi que les performances des oies (mortalité, faible poids de foie).

\section{3 / Effets de la nature physico- chimique des aliments}

Arroyo et al (2012c) ont montré qu'un changement de céréale (sorgho vs. maïs) entre les phases d'élevage et de gavage réduisaient les performances des animaux avec une augmentation de la mortalité et une réduction de poids de foie. Ces résultats peuvent être expliqués par la nécessité pour le tube digestif de s'adapter à un aliment de composition chimique différente malgré une composition nutritionnelle semblable. Ainsi, le maïs et le sorgho présentent des caractéristiques similaires concernant les macronutriments (teneurs en lipides, parois végétales, amidon et protéines ; Sauvant et al 2004). En revanche, il existe des différences fines entre ces deux céréales concernant les micronutriments ou la composition chimique des macronutriments. Par exemple, les protéines sont en partie spécifiques (zéine pour le maïs, kafirine pour le sorgho). De plus, le type d'amidon, principalement le rapport amylopectine/amylose, diffère entre les deux céréales (Jenkins et Donald 1995, D’Alfonso et
McCracken 2002). Or, il a été montré que chez les oiseaux, la digestibilité des amylopectines est plus élevée que celle de l'amylose (Skiba et al 2005, Zhou et al 2010). Ces données sont corroborées chez l'oie par des poids de foie plus élevés obtenus chez des animaux gavés avec des matières premières riches en amylopectines (du sorgho par rapport à du maïs corné denté ; Arroyo et al 2012c).

En conséquence, on peut recommander d'adapter les apports alimentaires à la composition nutritionnelle vraie des constituants de l'aliment et surtout d'incorporer la céréale qui sera utilisée pour le gavage des animaux dans l'aliment distribué au cours de la phase de «croissance-finition» afin d'adapter le tube digestif et de limiter les conséquences de ces transitions alimentaires entre phase de «croissance-finition» et phase de gavage.

\section{Conclusion}

En élevage d'oies pour la production de foie gras, la stratégie d'alimentation est orientée vers la préparation des animaux pour le gavage. En conséquence, les oies sont soumises tout au long de leur vie à des changements de forme et de composition de l'aliment et à des temps d'accès à la mangeoire qui évoluent au cours du temps. Ces changements ont pour objectif d'adapter leur alimentation à l'évolution de leurs besoins et de les préparer à la phase de gavage. Les transitions se traduisent fréquemment par une réduction transitoire de l'ingestion qui peut entraîner une hétérogénéité du poids et des performances des animaux au sein d'un groupe. Ces effets s'expliquent par le délai nécessaire à la mise en place des adaptations comportementale et digestive des animaux face aux modifications de l'aspect, de la taille des particules, de la nature chimique ou de la capacité de rétention en eau des aliments. Une meilleure prise en compte, dans la conduite alimentaire, des préférences alimentaires, des capacités d'apprentissage et des capacités digestives des oies, constitue un challenge ambitieux pour minimiser l'effet des transitions alimentaires sur les performances des animaux et maximiser la réussite du système d'élevage. 


\section{Références}

Amerah A.M., Ravindran V., Lentle R.G., Thomas D.G., 2007. Influence of feed particle size and feed form on the performance, energy utilization, digestive tract development, and digesta parameters of broiler starters. Poult. Sci., 86, 2615-2623.

Arroyo J., Auvergne A., Dubois J.P., Lavigne F., Bijja M., Fortun-Lamothe L., $2012 \mathrm{a}$. Influence of feeding sorghum on the growth, gizzard development and carcass traits of growing geese. Animal., 6, 1583-1589.

Arroyo J., Auvergne A., Dubois J.P., Lavigne F., Bijja M., Bannelier C., Fortun-Lamothe L., $2012 \mathrm{~b}$. Effects of presentation and type of cereals (corn or sorghum) on performance of geese. Poult. Sci., 91, 2063-2071.

Arroyo J., Auvergne A., Dubois J.P., Lavigne F., Bijja M., Bannelier C., Fortun-Lamothe L., 2012 c. Effects of substituting yellow corn by sorghum in goose diet on magret and foie gras quality. En préparation.

Auvergne A., Leprettre S., Lavigne F., Babilé R., Dubois J.P., 2006. Utilisation de protéagineux métropolitains dans des mélanges fermiers par les oies grises du sud-ouest en phase de finition. $7^{\text {mes }}$ Journ. Rech. Palmipèdes à Foie Gras. Arcachon, France, 4p.

Auvergne A., Lavigne F., Dubois JP., 2008a. Utilisation du maïs en alimentation fermière pour les oies en période de croissance-finition. $8^{\text {imes }}$ Journ. Rech. Palmipèdes à Foie Gras. Arcachon, France, $4 \mathrm{p}$.

Auvergne A., Tortereau F., Rémignon H., Lavigne F., Dubois J.P., 2008b. Effets des conditions d'éclosion et de démarrage sur le poids du pectoral à la mise en gavage et les résultats de gavage. $8^{\text {imes }}$ Journ. Rech. Palmipèdes à Foie Gras. Arcachon, France, 4p.

Auvergne A., Dubois J.P., Lavigne F., Bijja M., 2010. Effets des conditions de températures et du délai d'alimentation en démarrage sur les résultats d'élevage et de gavage des oies. $9^{\text {èmes }}$ Journ. Rech. Palmipèdes à Foie Gras. Bordeaux, France. 4p.

Auvergne A., Arroyo J., Bijja M., Lavigne F., Dubois J.P., 2012. Intérêt d'une alimentation multiphase pour l'élevage des oies destinées au gavage. Essais S107-S1013. Rapport d'étude CIFOG.

Babilé R., Chambert S., Everlet P., Auvergne A., Manse H., 1998. Caractérisation des tissus adipeux après gavage des canards au maïs ou au mélange figue-maïs. Proc. $3^{\text {èmes }}$ Journ. Rech. Palmipèdes à Foie Gras, Bordeaux, France, 133-135.

Bijja M., Dubois J.P., Lavigne F., Auvergne A., Arroyo J., Fernandez X., 2010. Qualité des parcours de palmipèdes: comparaison de la conduite en parcours tournants et en parcours fixe. $9^{\text {èmes }}$ Journ. Rech. Palmipèdes à Foie Gras, Bordeaux, France, 197-201.

Blundell J.E., 1991. Pharmacological approaches to appetite suppression. Trends Pharmacol. Sci., 12, 147-157.

Bouiller-Oudot M., Leprettre S., Dubois J.P., Babilé R., 2002. Utilisation du tourteau de noix en gavage : incidence sur les produits d'oies grasses. Proc. $5^{\text {emes }}$ Journ. Rech. Palmipèdes à Foie Gras, Pau, France, 132-135.

Bouvarel I., Vallée C., Lescoat P., Ferreira G., Chagneau A.M., Constantin P., Leterrier C.,
2008. Effects of various energy and protein levels on feed preferences in meat-type chickens used to sequential feeding. Animal, 2, 1674-1681.

Bouvarel I., Chagneau A.M., Lecuelle S. Lescoat P., Ferreira G., Duvaux-Ponter C., Leterrier C., 2009. Feed composition and hardness interact in preference and intake in chickens. Appl. Anim. Behav. Sci., 118, 62-68.

Bouvarel I., Tesseraud S., Leterrier C., 2010 L'ingestion chez le poulet de chair : n'oublions pas les régulations à court terme. INRA Prod. Anim., 23, 391-404.

Buffo R.A., Weller C.L., Parkhurst A.M., 1998. Relationships among grain sorghum quality factors. Cereal Chem., 75, 100-104.

Capretta P.J., Moore M.J., 1970. Appropriateness of reinforcement to cue in the conditioning of food aversions in chickens (Gallus gallus). J. Comp. Physiol. Psychol., 72, 85-89.

Carré B., 2000. Effets de la taille des particules alimentaires sur les processus digestifs chez les oiseaux d'élevage. In : Numéro spécial, Granulométrie des aliments. INRA Prod. Anim., 13, 131-136.

CIFOG (Comité Interprofessionnel des palmipèdes à Foie Gras), 2012. Rapport économique. Assemblée Générale du 22/06/2012. Saint Palais, France.

Clayton D.A., 1978. Social facilitated behaviour. Q. Rev. Biol., 53, 373-390.

Clemens E.T., Stevens C.E., Southwort M. 1975. Sites of organic acid production and pattern of digesta movement in the gastrointestinal tract of geese. J. Nutr., 105, 1341-1350.

Covasa M., Forbes J.M., 1994. The effect of social interaction on selection of feeds by broiler chickens. Br. Poult. Sci., 35, 817.

Daghir N.J., 2008. Poultry production in hot climates. In: Nuhad J. Daghir (Ed). $2^{\text {nd }}$ Edition, Published by CABI, Wallinford, Oxfordshire, UK, 387p.

D'Alfonso T.H., McCracken K., 2002. Global corn quality variability. Proc.MultiState Poultry Meeting, Indianapolis, Indiana, 14-16.

Dubois J.P., Guichard F., Auvergne A., Guy G., Babilé R., Leprettre S., Lavigne F., 1996a. Comparaison des performances de croissance et d'engraissement de trois types génétiques d'oies. $2^{\text {emes }}$ Journ. Rech. Palmipèdes à Foie Gras. Bordeaux, France, 5-8.

Dubois J.P., Auvergne A., Babilé R., Verdier M., Leprettre S., Lavigne F., Vieillecroze D. 1996b. Le point des facteurs d'amélioration de la production d'oies gavées. $2^{\text {èmes }}$ Journ. Rech. Palmipèdes à Foie Gras. Bordeaux, France, 89-92.

Dubois J.P., Leprettre S., Lavigne F., Babilé R., 2006. Conditions de démarrage des oisons. Détermination des besoins en température pendant la phase de démarrage. Incidence de la teneur énergétique de l'aliment. $7^{\text {èmes }}$ Journ. Rech. Palmipèdes à Foie Gras. Arcachon, France, 192-196.

Dubois J. P., Bijja M., Auvergne A., Lavigne F., Fernandez X., Babilé R., 2008. Qualité des parcours de palmipèdes : choix des espèces végétales, rendement et résistance au piétinement. $8^{\text {emes }}$ Journ. Rech. Palmipèdes à Foie Gras. Arcachon, France,107-110.
Dubois J.P., Auvergne A., Lavigne F., Fernandez X., Babile R., 2010. Le nouveau guide pratique du gavage. $9^{\text {emes }}$ Journ. Rech. Palmipèdes à Foie Gras. Bordeaux, France, 255-258.

Duke G.E., 1982. Gastrointestinal motility and its regulation. Poultr. Sci., 61, 1245-1256.

Duke G.E., 1992. Recent studies on regulation of gastric motility in turkeys. Poultr. Sci., $71,1-8$.

Dunning J.B., 1992. CRC handbook of avian body masses, Boca Raton, CRC Press, 19.

Emmans G.C., 1991. Diet selection by animals: theory and experimental design. Proc. Nutr. Soc., 50, 59-64.

Farell D., 2004. Management, nutrition and products of domestic geese: a review. Proc. Aust. Poult. Sci. Sym., 139-144.

Forbes J.M., Covasa M., 1995. Application of diet selection by poultry with particular reference to whole cereals. World's Poultr. Sci. J., 51, 149-165.

Forbes J.M., Shariatmadari F., 1994. Diet selection for protein by poultry. World's Poult. Sci. J., 50, 7-24.

Giger-Reverdin S., 2000. Charaterisation of feedstuffs for ruminants using some physical parameters. Anim. Feed Sci. Technol., 86, 5369.

Guéméné D., Guy G., 2004. The past, present and future of force-feeding and "foie gras" production. World's Poultr. Sci. J., 60, 210222.

Guy G., Buckland R., 2002. Goose Production. FAO Animal Production and Health Paper, Rome, 154

Guy G., Baéza E., Salichon M.R., Juin H., Rousselot-Pailley D., 1998. Influence des conditions d'élevage de l'oie sur la production de foie gras et de viande. Ann. Zootech., 47, 215-224.

Henuk Y.L., Dingle J.G., 2002. Practical and economic advantage of choice feeding systems for laying poultry. World's Poult. Sci. J., 58, 199-208.

Hermier D., Salichon M.R., Guy G., Peresson R., Mourot J., Lagarrigue S., 1999. La stéatose hépatique des palmipèdes gaves : bases métaboliques et sensibilité génétique. In : Numéro spécial, Lipogenèse et qualité des produits animaux. Chilliard Y. (Ed). INRA Prod. Anim., 12, 265-271.

Hsu J.C., Lu T.W., Chiou P.W.S., Yu B., 1996. Effects of different sources of fibre on growth performance and apparent digestibility in geese. Anim. Feed Sci. Tech., 60, 93-102.

Hughes B.O., 1971. Allelomimetic feeding in the domestic fowl. Br. Poult. Sci., 12, 359366 .

Hughes B.O., 1984. The principles underlying choice feeding behavior in fowls with special reference to production experiments. World's Poult. Sci. J., 40, 141-150.

Jamroz D., Jakobsen K., Orda J., Skorupiska J., Wiliczkiewicz A., 2001. Development of gastrointestinal tract and digestibility of dietary fibre and amino acids in young chickens, ducks and geese fed diets with high 
amounts of barley. Comp. Biochem. Physiol., A, 131, 657-668.

Jenkins P.J., Donald A.M., 1995. The influence of amylose on starch granule structure. Int. J. Biol. Macromol., 17, 315-321.

Keeling L.J., Hurnik J.F., 1996. Social facilitation acts more to the appetitive than the consummatory phase of feeding behavior in domestic fowl. Anim. Behav., 52, 11-15.

Kozak J., Bodi L., Janan I., Karasi M., 1997. Improvements in the reproductive characteristics of hungarian upgraded and grey lands geese in Hungary. World's Poult. Sci. J., 53, 197-201.

Lavigne F., Dubois J.P., Leprettre S., 2000. Guide pratique du gavage des oies: normes

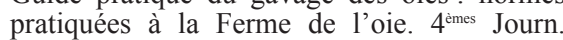
Rech. Palmipèdes à Foie Gras. Arcachon, France, 117-120.

Leclercq B., Blum J.C., Sauveur B., Stevens P., 1987. Nutrition of geese. In: Feeding of non-ruminants livestock, Butterworths, Londres, UK., 110-112.

Lecuelle S., Bouvarel I., Chagneau A.M., Lescoat P., Laviron F., Leterrier C., 2010. Feeding behaviour in turkeys with a changeover from crumbs to pellets. Appl. Anim. Behav. Sci., 125, 132-142.

Leeson S., Summers J.D., 1997. Feeding programs for waterfowl. In: Commercial Poultry Nutrition. Second edition, Ontario, University Books, 332-340.

Leprettre S., Babilé R., Auvergne A., Dubois J.P., Manse H., Verdier M., 1997. Feed restriction in Landese geese breeding: influences on growth and carcass composition during the growing period and after force-feeding. $11^{\text {th }}$ Eur. Waterfowls Symp., World Poultry Sci. Assoc., Nantes, France, 5 p.

Leprettre S., Dubois J.P., Lavigne F., Babilé R., 2000a. Système d'élevage des oies et performances de croissance. $4^{\text {èmes }}$ Journ. Rech. Palmipèdes à Foie Gras. Arcachon, France. 4p.

Leprettre S., Dubois J.P., Lavigne F., Babilé R., 2000b. Densité en élevage et performances de croissance chez l'oie destinée au gavage. $4^{\text {èmes }}$ Journ. Rech. Palmipèdes à Foie Gras. Arcachon, France, 4p.

Lu J., Kong X.L., Wang Z.Y., Yang H.M., Zhang K.N., Zou J.M., 2011. Influence of whole corn feeding on the performance, digestive tract development, and nutrient retention of geese. Poult. Sci., 90, 587-594.
Marcilloux J.C., Auffray P., 1981. Détermination du profil alimentaire de l'oie de race landaise. Reprod. Nutr. Dev., 737-748.

Meltzer A., 1987. Acclimatization to ambient temperature and its nutritional consequences. World's Poultry Sci. J., 43, 33-44.

Mirghelenj S.A., Golian A., 2009. Effects of feed form on development of digestive tract, performance and carcass traits of broiler chickens. J. Anim. Vet. Adv., 8, 1911-1916.

Moore S.J., 1999. Food breakdown in an avian herbivore: who needs teeth? Aust. J. Zool., 47, 625-632.

Nielsen B.L., 2004. Behavioural aspects of feeding constraints: do broilers follow their gut feeling? Appl. Anim. Behav. Sci., 86, 251-260.

Nir I., Perek M., Katz Z., 1972. The influence of soybean meal supplemented to maize diet of forced-fed geese upon their liver, organ and blood plasma components. Ann. Biol. Anim. Bioch. Biophys., 12, 77-89.

Nitsan Z., Dvorin A., Nir I., 1981. Composition and amino acid content of carcass, skin and feathers of the growing gosling. Br. Poult. Sci., 22, 79-84.

Noirot V., Bouvarel I., Barrier-Guillot B., Castaing J., Zwick J.L., Picard M., 1998. Céréales entières pour le poulet de chair : le retour? INRA Prod. Anim., 11, 349-357.

NRC (National Research Council), 1994. Nutrient requirements of geese. In: Nutrient Requirements of Poultry, $9^{\text {th }}$ revised Edition. National Academic Press, Washington, USA, 40-41.

Pinnavaia G.G., Pizzanrani S., 1998. Evaluation of the degree of gelatinization by water holding capacity. Starch., 50, 64-67.

Pousga S., Boly H., Ogle B., 2005. Choice feeding of poultry: a review. Livest. Res. Rural Dev., 17, 4 .

Rameseyer A., Petit O., Thierry B., 2009. Décision-making in group departures of females domestic geese. Behaviour, 146, 351371 .

Robertson J.A., Eastwood M.A., 1981. An investigation of the experimental conditions which could affect water-holding capacity of dietary fibre. J. Sci. Food Agric., 32, 819825 .

Romanov M.N., 1999. Goose production efficiency as influenced by genotype, nutrition and production systems. World's Poultr. Sci. J., $55,281-294$.
Roper T.J., Marples N.M., 1997. Colour preferences of domestic chicks in relation to food and water presentation. Appl. Anim. Behav. Sci., 54, 207-213.

Roussely M., 2000. Synthèse sur les performances techniques des souches d'oies pour la production de foie gras. $4^{\text {emes }}$ Journ. Rech. sur les Palmipèdes à Foie Gras, Arcachon, France, 9-12.

Rouvier R., Poujardieu B., Rousselot-Pailley D., Larrue P., Esteve D., 1992. Paramètres génétiques des caractères de croissance, de gavage et de foie gras dans le croisement de deux souches d'oies (Anser anser) sélectionnées. Genet. Sel. Evol., 24, 53-69.

Sauvant D., Perez J.M., Tran G., 2004. Tables de composition et de valeur nutritive des matières premières destinées aux animaux d'élevage: porcs, volailles, bovins, ovins, caprins, lapins, chevaux, poissons. $2^{\text {eme }}$ Edition revue et corrigée. INRA Editions, Paris, France, 301p.

Savory C.J., 1999. Temporal control of feeding behaviour and its association with gastrointestinal function. J. Exp. Zool., 283, 339-347.

Skiba F., Barrier-Guillot B., Metayer J.P., Champion M., 2005. Effet du type de maïs et du type d'amidon sur la valeur alimentaire du maïs pour le poulet de chair. $6^{\text {emes }}$ Journ. Rech. Avicole. St Malo, France, 4p.

Sturkie P.D., 1986. Avian Physiology. Fourth edition. Springer-Verlag, New York.

Umar Faruk M., Bouvarel I., Meme M., Rideau N., Roffidal L., Tukur H.M., Bastianelli D., Nys Y., Lescoat P., 2010. Sequential feeding using whole wheat and a separate proteinmineral concentrate improved feed efficiency in laying hens. Poult. Sci., 89, 785-796.

Yang H.M., Wang Z.Y., Wang J., Shi S.R., Zhu X.H., 2009. Effects of caecectomy on digestibility of crude protein, calcium, phosphorus, neutral detergent fibre and acid detergent fibre in geese. Arch. Geflugelk., 73,189192.

Yo T., Siegel P.B., Guerin H., Picard M., 1997. Self-selection of dietary protein and energy by broilers grown under a tropical climate: effect of feed particle size on the feed choice. Poult. Sci., 76, 1467-1474.

Zhou Z., Wan H.F., Li Y., Chen W., Qi Z.L., Peng P., Peng J., 2010. The influence of the amylopectin/amylose ratio in samples of corn on the true metabolizable energy value for ducks. Anim. Feed Sci. Technol., 157, 99103.

\section{Résumé}

Le mode de conduite alimentaire des oies destinées à la production de foie gras nécessite de nombreux changements de rythme d'ingestion et de nature d'aliments. L'objectif de cette synthèse est, après avoir présenté l'anatomie, les besoins nutritionnels, la croissance et la conduite alimentaire, d'identifier les causes et les solutions envisageables pour affronter ces périodes sensibles. En effet, afin de préparer les oies à recevoir une forte quantité d'aliments dans une période courte (gavage), les oies sont soumises tout au long de leur vie à des changements d'aliments et de rythmes d'accès aux mangeoires afin d'augmenter leur capacité d'ingestion. Lors de chacune de ces phases de transitions, on observe fréquemment des baisses de consommation, ce qui entraine une hétérogénéité entre les lots et une réduction des performances des animaux. La gestion de ces transitions vient se confronter aux capacités d'adaptation comportementale et digestive des animaux face à des modifications des caractéristiques sensorielles et physico-chimiques des aliments : couleur, texture, taille des particules, capacité de rétention hydrique, composition nutritionnelle dont la nature de l'amidon (ratio amylopectine/amylose). La gestion de ces transitions consiste à trouver un compromis entre les préférences alimentaires des animaux et leur préparation à la période de gavage pour la production de foie gras. 


\begin{abstract}
Schedule and management of food transitions in geese for the production of "foie gras"

The feeding schedule of geese for the production of "foie gras" requires many changes of ingestion rhythms and the nature of food. The aim of this review was, after presenting the anatomy, nutritional requirements, growth and dietary behavior of the animals, to identify the causes and possible solutions needed for the overfeeding period. Indeed, in order to prepare the geese to receive a large amount of food in a short period (overfeeding period), the geese are subjected throughout their life to different feed changes and access time to feeders in order to increase their intake capacity. At each transition phase, reductions in consumption are frequently observed, which leads to a heterogeneity between batches and to reduced animal performance. The management of these transitions has to confront the behavioral and digestive adaptation capacities of the animals when they are faced with changes in sensory and physico-chemical characteristics of food: color, texture, particle size, water retention, nutrient composition, nature of starch (amylopectin / amylose). The management of these transitions consists in finding a compromise between the food preferences of animals and their preparation for the overfeeding period for "foie gras" production.
\end{abstract}

ARROYO J., FORTUN-LAMOTHE L., DUBOIS J.-P., LAVIGNE F., AUVERGNE A., 2012. Conduite et gestion des transitions alimentaires chez les oies destinées à la production de foie gras. INRA Prod. Anim., 25, 5, 419-430. 